\title{
TITLE:
}

\section{Evolution of cosmological perturbations in the brane world}

$\operatorname{AUTHOR}(S):$

Koyama, K; Soda, J

CITATION:

Koyama, K ... [et al]. Evolution of cosmological perturbations in the brane world. PHYSICAL REVIEW D 2000, 62(12): 123502.

ISSUE DATE:

2000-12-15

URL:

http://hdl.handle.net/2433/49959

RIGHT:

Copyright 2000 American Physical Society 
PHYSICAL REVIEW D, VOLUME 62, 123502

\title{
Evolution of cosmological perturbations in the brane world
}

\author{
Kazuya Koyama \\ Graduate School of Human and Environmental Studies, Kyoto University, Kyoto 606-8501, Japan \\ Jiro Soda \\ Department of Fundamental Physics, FIHS Kyoto University, Kyoto 606-8501, Japan
}

(Received 30 May 2000; published 14 November 2000)

\begin{abstract}
The evolution of cosmological perturbations is studied in the context of the Randall-Sundrum brane world scenario, in which our universe is realized on a three-brane in five-dimensional anti-de Sitter (AdS) spacetime. We develop a formalism to solve the coupled dynamics of the cosmological perturbations in the brane world and the gravitational wave in the AdS bulk. Using our formalism, the late time evolution of the cosmological scalar perturbations at any scales larger than the AdS curvature scale $l$ is shown to be identical to the one obtained in the conventional 4D cosmology, provided the effect of heavy graviton modes may be neglected. Here the late time means the epoch when the Hubble horizon $H^{-1}$ in the 4D brane world is sufficiently larger than the AdS curvature scale $l$. If the inflation occurs sufficiently lower than $l^{-1}$, the scalar temperature anisotropies in the cosmic microwave background at large scales can be calculated using the constancy of the Bardeen parameter as is done in the 4D cosmology. The assumption of the result is that the effect of the massive graviton with mass $m e^{-\alpha_{0}}>l^{-1}$ in the brane world is negligible, where $e^{\alpha_{0}}$ is the scale factor of the brane world. We also discuss the effect of these massive gravitons on the evolution of the perturbations.
\end{abstract}

PACS number(s): $98.80 . \mathrm{Cq}$

\section{INTRODUCTION AND SUMMARY}

Much attention has been paid to the possibility that we are living on a 3-brane in higher dimensional spacetime [1,2]. This brane world picture alters the conventional notion of extra dimensions. Particularly if the bulk is anti-de Sitter (AdS) spacetime, the extra dimensions could be large or even infinite. The action describing the brane world picture is given by

$$
\begin{aligned}
S= & \frac{1}{2 \kappa^{2}} \int d^{5} x \sqrt{-g}\left(\mathcal{R}^{5}+\frac{12}{l^{2}}\right) \\
& -\sigma \int d^{4} x \sqrt{-g_{\text {brane }}}+\int d^{4} x \sqrt{-g_{\text {brane }}} \mathcal{L}_{\text {matter }},
\end{aligned}
$$

where $\mathcal{R}^{5}$ is the 5D Ricci scalar, $l$ is the curvature radius of the AdS spacetime and $\kappa^{2}=8 \pi G$ where $G$ is the Newton constant in the 5D spacetime. The brane has tension $\sigma$ and the induced metric on the brane is denoted as $g_{\text {brane }}$. Matter is confined to the 4D brane world and is described by the Lagrangian $\mathcal{L}_{\text {matter }}$. We will assume $Z_{2}$ symmetry across the brane.

Recently, Randall and Sundrum (RS) constructed a simple model for a brane world [3]. They assumed the effect of the matter confined to the brane is negligible compared with that of the surface tension. Their solution is described by the metric

$$
d s^{2}=\left(\frac{l}{z}\right)^{2}\left(d z^{2}-d \tau^{2}+\delta_{i j} d x^{i} d x^{j}\right)
$$

It has been shown that the usual 4D gravitational interactions are recovered on the 3-brane. One of the fascinating features of their model is that 5D spacetime is not necessarily compactified.

In the RS model, the 3-brane is Minkowski spacetime. Solutions for homogeneous expanding brane world are obtained by many people [4-14]. It has been shown that the evolution of the universe is identical to that of the conventional 4D cosmology at sufficiently low energies. However, in the real world, the universe has inhomogeneity which leads to our structure of the universe [15-17]. This inhomogeneity can be observed today, for example, in the cosmic microwave background radiation (CMB). Then the cosmological perturbations in the brane world give direct tests for a viability of the brane world idea. In addition, the inhomogeneous fluctuations on the brane could be a powerful observable to probe the existence of the extra dimensions. This is because the inhomogeneous fluctuations on the brane inevitably produce the perturbations of the bulk geometry [18]. The perturbations in the bulk affect the motion of the brane in turn. Then, in general, the dynamics on the brane cannot be separated from the dynamics in the bulk. This could add a new property to the evolution of the cosmological perturbations and could reject the brane world idea.

To study the evolution of cosmological perturbations, we should treat the coupled system of brane-bulk dynamics. The problem has a similarity with the dynamics of the domain wall interacting with the gravitational wave, which has been investigated in 4D spacetime [19]. In our case, the matter on the brane is dynamical. This makes the problem very difficult. We should find a solution for the brane with the cosmological expansion and inhomogeneous fluctuations. The most straightforward way is to solve the 5D Einstein equation, however, it would be difficult to carry out in general.

In this paper we propose a new method to deal with the problem. We observe that the brane world cosmology can be 
constructed by cutting the perturbed AdS spacetime along the suitable slicing and gluing two copies of remaining spacetime. The point is as follows. If we choose a slicing to cut the 5D AdS spacetime, the jump of the extrinsic curvature along the slicing is determined. Because the jump of the extrinsic curvature should be equated with the matter localized on the brane, the matter on the brane is also determined. In other words, a solution for a brane with the given matter can be obtained by finding a suitable slicing. To find the suitable slicing for the given matter, we need two kinds of coordinate transformations. One is a large coordinate transformation which leads to the slicing which determines the background matter. Another is an infinitesimal coordinate transformation which leads to the slicing which determines matter perturbations. The coordinate transformations will be determined by imposing the conditions on the matter such as equation of state. More detailed procedures will be described in the next section.

Our main result is

$$
\frac{\delta \rho}{\rho}=-2 \Phi_{0}=\mathrm{const}
$$

at superhorizon scales and for late times when the Hubble scale $H$ is sufficiently low $\left(H \ll l^{-1}\right)$. Here $\delta \rho$ is the density fluctuations and $\Phi_{0}$ is the metric perturbations in the longitudinal gauge in the brane world and we assumed the barotropic index of the matter is constant. The point to observe is that the solution (3) is identical to the one obtained in the 4D cosmology. We can also show that the late time evolution of the perturbations agrees with the one obtained in the 4D cosmology at subhorizon scales larger than the AdS curvature scale $l$.

The assumption to obtain the above results is that the effect of the massive graviton with mass $m>m_{e f f}=l^{-1} e^{\alpha_{0}}$ in the brane world is negligible where $e^{\alpha_{0}}$ is the scale factor of the brane world. We can understand the fact that the massive graviton with $m>m_{\text {eff }}$ can modify the evolution from the following arguments. For late times, the cosmological brane approaches to the RS brane. For the RS brane, the 4D gravity is recovered by the zero-mode of $5 \mathrm{D}$ graviton $[3,20-$ 23]. The Kaluza-Klein modes give the correction to the 4D gravity. However, in the anti-de Sitter spacetime, the brane is protected from the Kaluza-Klein modes by the potential barrier which arises from the curvature of the AdS spacetiem. For earlier times, the cosmological brane is located at larger $z$ in the coordinate (2). The point is that, for larger $z$, the potential barrier becomes lower. Then the relatively light graviton can interact with the brane. Thus for early times, the 4D cosmology will be susceptible to the Kaluza-Klein modes of $5 \mathrm{D}$ graviton. If the brane interacts with the $5 \mathrm{D}$ gravitational perturbations, the gravitational waves are inevitably emitted to the 5D bulk. It will cause the modification in the evolution of the perturbations. This picture is consistent with the result that the modes with large $m>m_{e f f}=l^{-1} e^{\alpha_{0}}$ can modify the evolution because $m_{\text {eff }}$ becomes smaller for earlier times.

The paper is organized as follows. In Sec. II, we describe our formalism in detail and derive the background solution

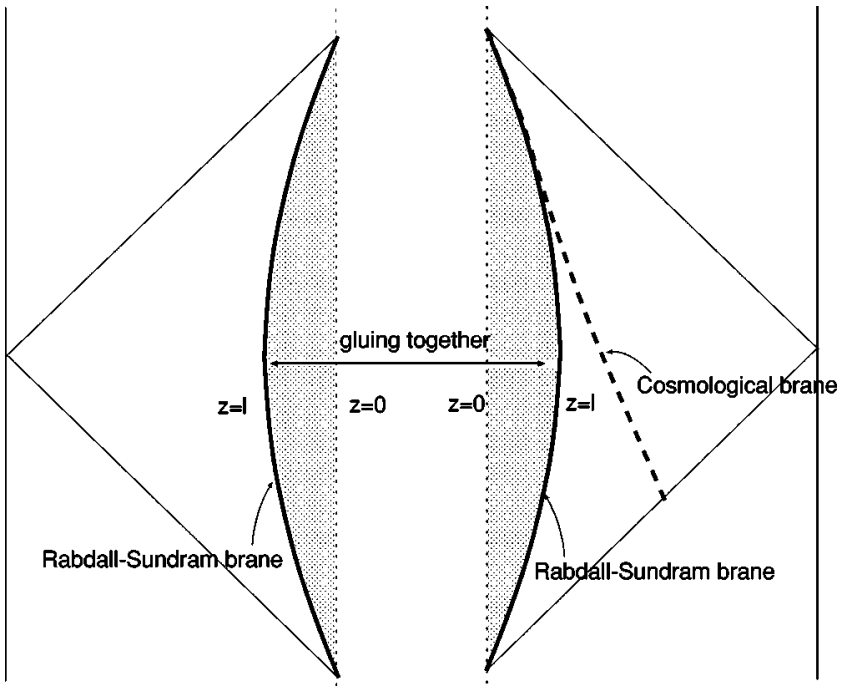

FIG. 1. Conformal diagram of the AdS spacetime. The thick line shows the trajectory of the RS brane and the dotted line shows the brane with cosmological expansion. The RS solution is obtained by deleting the AdS spacetime from $z=l$ to the boundary $z=0$ (shaded region) and gluing two copies of the remaining spacetime.

using it. In Sec. III, we calculate the perturbations at superhorizon scales for late times $H<l^{-1}$ using the formalism. In Sec. VI, we calculate the perturbations at subhorizon scales for late times $H<l^{-1}$. It will be shown that the evolution of the perturbations is identical with the one obtained in the 4D cosmology for any scales larger than the AdS curvature scale, if the effect of the massive graviton $m>m_{\text {eff }}$ is negligible. In Sec. V we study the effect of the massive graviton $m>m_{\text {eff }}$ on the evolution of the perturbations. Finally we discuss the implication of our results on the brane world cosmology. In the Appendix, we listed useful formulas for calculations.

\section{FORMALISM}

\section{A. Background}

We shall start with the Randall and Sundrum solution for the brane world [3]. They considered a single brane with positive tension $\sigma$ in the 5D anti-de Sitter spacetime. Setting the surface tension of the brane by

$$
\kappa^{2} \sigma=\frac{6}{l},
$$

and assuming the $Z_{2}$ symmetry across the brane, they found a solution described by the metric (2). The brane is located at $z=l$ (see Fig. 1). From the metric (2), we see the brane world is Minkowski spacetime.

Next we will seek the brane world with the cosmological expansion. For this purpose, we note that the RS solution can be obtained by the following procedure. First cut the AdS spacetime along $z=l$ and delete the AdS spacetime from $z$ $=l$ to the boundary $z=0$. Next glue two copies of the remaining spacetime along $z=l$ (see Fig. 1). The jump of the extrinsic curvature at $z=l$ should be equated with the matter 


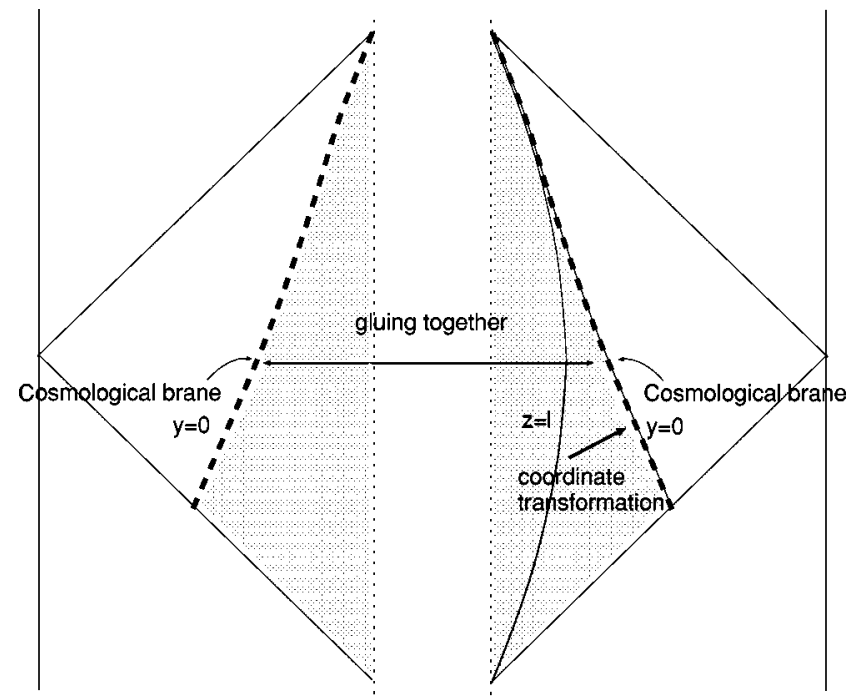

FIG. 2. The solution for a brane with cosmological expansion is obtained by deleting the AdS spacetime from $y=0$ to the boundary (shaded region) and gluing two copies of the remaining spacetime.

at $z=l$. Thus we must put the suitable matter on the brane to glue the spacetime. Then the content of the matter on the brane is restricted as Eq. (4). The above argument implies that if we use the different slicing to cut the AdS spacetime, we need different matter to glue the spacetime. This is because the jump of the extrinsic curvature depends on the slicing we use to cut the AdS spacetime. Thus if we can find appropriate slicing to cut the AdS spacetime, we can put suitable matter resulting the cosmological expansion on the brane (see Fig. 2).

Now, we explicitly carry out the procedure to find the appropriate slicing.

(1) Start with the AdS spacetime:

$$
d s^{2}=\left(\frac{l}{z}\right)^{2}\left(d z^{2}-d \tau^{2}+\delta_{i j} d x^{i} d x^{j}\right)
$$

(2) Make the coordinate transformation from the coordinate system $\left(z, \tau, x^{i}\right)$ to $\left(y, t, x^{i}\right)$ by

$$
z=l(f(u)-g(v)), \quad \tau=l(f(u)+g(v)),
$$

where $u, v$ are the null coordinates of the new coordinate system; $u=(t-y) / l, v=(t+y) / l$ and $f(u)$ and $g(v)$ are the arbitrary functions. The resulting metric is

$$
\begin{aligned}
d s^{2}= & 4 \frac{f^{\prime}(u) g^{\prime}(v)}{(f(u)-g(v))^{2}}\left(d y^{2}-d t^{2}\right) \\
& +\frac{1}{(f(u)-g(v))^{2}} \delta_{i j} d x^{i} d x^{j},
\end{aligned}
$$

where $f^{\prime}(x)=d f(x) / d x$ and $g^{\prime}(x)=d g(x) / d x$. For future convenience, we put

$$
e^{2 \beta(y, t)}=4 \frac{f^{\prime}(u) g^{\prime}(v)}{(f(u)-g(v))^{2}}, \quad e^{2 \alpha(y, t)}=\frac{1}{(f(u)-g(v))^{2}} .
$$

(3) Delete the AdS spacetime from $y=0$ to the boundary and glue two copies of remaining spcaetime. The jump of the extrinsic curvature at the brane $(y=0)$ is determined by the first derivative of the metric with respect to $y$ :

$$
\begin{aligned}
\alpha_{1}(t)= & \frac{1}{l} \frac{f^{\prime}(t / l)+g^{\prime}(t / l)}{f(t / l)-g(t / l)}, \\
\beta_{1}(t)= & \frac{1}{l}\left(\frac{f^{\prime}(t / l)+g^{\prime}(t / l)}{f(t / l)-g(t / l)}\right. \\
& \left.+\frac{1}{2} \frac{-f^{\prime \prime}(t / l) g^{\prime}(t / l)+f^{\prime}(t / l) g^{\prime \prime}(t / l)}{f^{\prime}(t / l) g^{\prime}(t / l)}\right),
\end{aligned}
$$

where we denote the power series expansion near the brane as

$$
\alpha(y, t)=\alpha_{0}(t)+\alpha_{1}(t)|y|+\frac{\alpha_{2}(t)}{2} y^{2} \cdots
$$

The jump of the extrinsic curvature should be equated with matter on the brane. Taking the 5D energy momentum tensor as

$$
T_{N}^{M}=\operatorname{diag}(0,-\rho, p, p, p) \delta(y),
$$

the junction condition can be read off as (see Appendix A)

$$
\begin{aligned}
& \alpha_{1}(t)=-\kappa^{2} e^{\beta_{0}}\left(\frac{\sigma}{6}+\frac{\rho(t)}{6}\right), \\
& \beta_{1}(t)=-\kappa^{2} e^{\beta_{0}}\left(\frac{\sigma}{6}-\frac{\rho(t)}{3}-\frac{p(t)}{2}\right) .
\end{aligned}
$$

(4) Determine the matter content on the brane by imposing the equation of state

$$
p=w \rho .
$$

Then it gives one constraint on $f$ and $g$. There remains one freedom in $f$ and $g$. Since $e^{\beta_{0}(y=0, t)}$ determines the time slicing in the brane world, it is a gauge freedom in the brane world. We fix the gauge degree of freedom by demanding that $t$ is the cosmological time,

$$
e^{2 \beta_{0}}=4 \frac{f^{\prime}(t / l) g^{\prime}(t / l)}{(f(t / l)-g(t / l))^{2}}=1
$$

Combining Eqs. (13) and (14), we can determine the function $f(t)$ and $g(t)$. The $y$ dependence of the metric can be obtained automatically by replacing $f(t)$ to $f(u)$ and $g(t)$ to $g(v)$. Hence we obtained the coordinate transformation which leads to a brane with matter of given equation of state.

The induced metric on the brane becomes 


$$
d s^{2}=-d t^{2}+e^{2 \alpha_{0}(t)} \delta_{i j} d x^{i} d x^{j}
$$

where $e^{\alpha_{0}(t)}=[f(t / l)-g(t / l)]^{-1}$ is the scale factor of the brane world. From Eqs. (13) and (14), we can verify that $\alpha_{0}$ and $\rho$ satisfy

$$
\begin{gathered}
\dot{\rho}+3 \dot{\alpha}_{0}(\rho+p)=0, \\
\dot{\alpha}_{0}^{2}=\frac{8 \pi G_{4}}{3} \rho+\frac{\kappa^{4} \rho^{2}}{36},
\end{gathered}
$$

where $\kappa^{4} \sigma=48 \pi G_{4}$. The former is the usual conservation of the energy. Since the term proportional to $\rho^{2}$ falls rapidly, the latter is identical with the Friedmann equation for late times. We show the solution of $f(u)$ and $g(v)$ for late times in Appendix A. The solution has two constants of the integration. We will normalize the scale factor as $e^{\alpha_{0}(t=t}$ present $)$ $=1$.

\section{B. Perturbations}

In the previous subsection, we obtained the brane world with the cosmological expansion. In the real world the universe has inhomogeneity which leads to our structure of the universe. Then it is important to obtain the solution for an inhomogeneous brane world. In this paper, we will concentrate our attention to the scalar perturbations. Unlike the homogeneous brane, we cannot place the inhomogeneous brane in the exact AdS spacetime as is shown in Ref. [18]. This is because the inhomogeneous perturbations in the brane world inevitably produces the perturbations in the geometry of the bulk. The perturbations in the bulk affects the motion of the brane in turn. Then the equations for metric perturbations and matter perturbations in the brane world cannot be separated from the dynamics in the bulk. We should solve the 5D perturbations at the same time. The coupled equations for the brane dynamics and gravitational perturbations in the bulk are in general very difficult to deal with.

The nonseparable nature of the brane-bulk dynamics can be seen from the power series expansion of the 5D Einstein equation near the brane. We will denote the power series expansion near the brane as in Eq. (10). The dynamical variables of the brane are the potential perturbations $\Phi_{0}$, curvature perturbations $\Psi_{0}$, density perturbations $\delta \rho$ and velocity perturbations $v$ (see Appendix B). We have two equations from conservations of energy-momentum tensor $T_{\mu}^{\mu \nu}=0$,

$$
\begin{gathered}
\dot{\delta} \rho=(\rho+p)\left(3 \dot{\Psi}_{0}+e^{-\alpha_{0}} \nabla^{2} v\right) \\
-3 \dot{\alpha}_{0}(\delta \rho+\delta p) \\
\left((\rho+p) e^{\alpha_{0} v}\right)=-3 \dot{\alpha}_{0} e^{\alpha_{0}} v+\delta p+(\rho+p) \Phi_{0},
\end{gathered}
$$

and the trace part of the Einstein equation in the brane world from 5D Einstein equation:

$$
\begin{gathered}
\ddot{\Psi}_{0}+4 \dot{\alpha}_{0} \dot{\Psi}_{0}+\dot{\alpha}_{0} \dot{\Phi}_{0}+2\left(\ddot{\alpha}_{0}+2 \dot{\alpha}_{0}^{2}\right) \Phi_{0} \\
-\frac{1}{3} e^{-2 \alpha_{0}}\left(2 \nabla^{2} \Psi_{0}-\nabla^{2} \Phi_{0}\right) \\
=\frac{\kappa^{2}}{3}\left(\frac{\beta_{1}}{2} \delta \rho-\frac{3 \alpha_{1}}{2} \delta p\right)
\end{gathered}
$$

In the conventional 4D cosmology, in addition to these equations, we have the equation

$$
\Phi_{0}-\Psi_{0}=0
$$

for matter with no anisotropic stress. Then we have closed set of the equations. However in the brane world, the correspondent equation derived from the 5D Einstein equation is

$$
E_{2}=-e^{-2 \alpha_{0}}\left(\Phi_{0}-\Psi_{0}+N_{0}\right)
$$

where $E$ is the nondiagonal $(i, j)$ component and $N$ is the $(y, y)$ component of the metric perturbations. The equation contains $E_{2}$, so we cannot have closed set of equations for the variables on the brane. This is because the inhomogeneous fluctuations on the brane inevitably produces the gravitational wave in the bulk, which gives the effective anistoropic stress to the perturbations.

However, the procedure to obtain the background solution can be applied for the inhomogeneous brane. The strategy is as follows. We first consider the perturbed 5D AdS spacetime in the coordinate system (5). We assume the perturbations are small enough to treat by linear perturbations. Because there is no matter in the bulk, the perturbations should satisfy the vacuum wave equation in the bulk. In the coordinate system (2), the wave equation can be solved easily with the help of the transverse-traceless (TT) gauge. In the 5D spacetime, the free graviton has five independent components which include one scalar component. Thus there is one variable for the choice of the perturbed AdS spacetime. Next, we take the coordinate transformation (6) to provide the cosmological background. The transformation function $f(u)$ and $g(v)$ is determined by the background matter. The perturbations in the coordinate system $\left(y, t, x^{i}\right)$ is then easily obtained by the usual procedure of the coordinate transformation.

Once the perturbed AdS spacetime is obtained, one might attempt to cut the spacetime along $y=0$ and glue two copies of remaining spacetime as is done for the background spacetime. However, we need to be more careful. The presence of matter on the brane bends the brane. For the background matter we made coordinate transformation so that the brane is located at $y=0$. However the matter perturbations also bends the brane. Then the perturbed brane is no longer located at $y=0$ (see Fig. 3). The perturbations evaluated at $y$ $=0$ is not the perturbations induced on the brane. Since the observers in the brane world are confined to the brane, we should evaluate the perturbations induced on the brane. Thus we should make (infinitesimal) coordinate transformation $\bar{x}^{M}=x^{M}+\xi^{M}$ to ensure that $\bar{y}=0$ denotes the location of the brane. In general, the coordinate transformation makes $g_{y \mu}\left(\mu=t, x^{i}\right)$ nonzero. These components can be gauged 


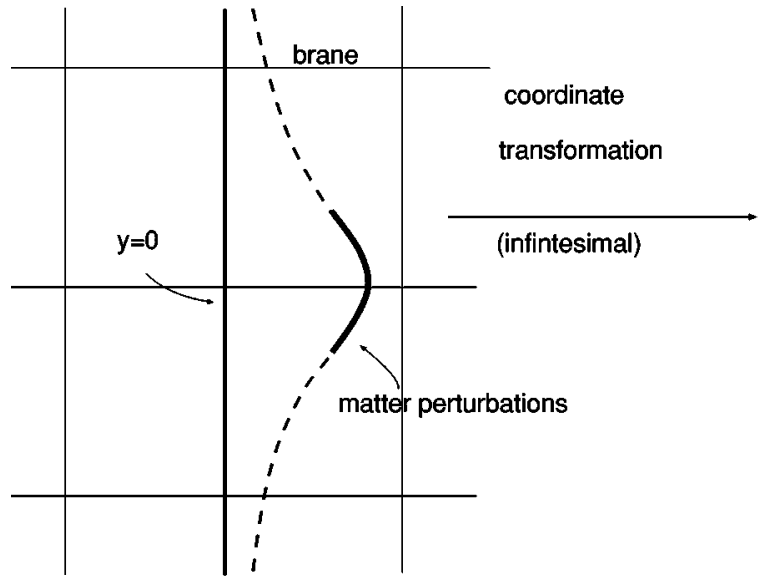

away, so we will take $g_{y \mu}=0$. Now the gluing can be performed so as to determine the cosmological perturbations.

Then we impose the conditions on the matter perturbations to determine the variable for the choice of the perturbed AdS spacetime and the infinitesimal coordinate transformation. The point is that imposing the conditions on the matter is equivalent to solving the Einstein equation. Let us remember that we impose the equation of state $p=w \rho$ (13) on the matter in deriving the background solution, which gives the Friedmann equation and conservation of the energy on the brane (16). The same is true of the perturbations.

We summarize the procedure to obtain the inhomogeneous brane.

(1) Let us start with the AdS spacetime with linear scalar perturbations:

$$
\begin{aligned}
d s^{2}= & \left(\frac{l}{z}\right)^{2}\left(d z^{2}-(1+2 \phi) d \tau^{2}+2 b_{, i} d x^{i} d \tau\right. \\
& \left.+\left[(1-2 \hat{\Psi}) \delta_{i j}+2 \hat{E}_{, i j}\right] d x^{i} d x^{j}\right),
\end{aligned}
$$

where $b_{, i}$ denotes $\partial b / \partial x^{i}$ and $E_{, i j}$ denotes $\partial^{2} E / \partial x^{i} \partial x^{j}$. The independent component of the scalar $5 \mathrm{D}$ graviton is one. We will use the transverse-traceless gauge to fix the gauge freedom. The gauge fixing conditions are given by

$$
\begin{aligned}
\phi-3 \hat{\Psi}+\nabla^{2} \hat{E} & =0, \\
2 \dot{\phi}+\nabla^{2} b & =0, \\
\dot{b}+2 \hat{\Psi}-2 \nabla^{2} \hat{E} & =0 .
\end{aligned}
$$

Using these conditions, the Einstein equation in the bulk becomes

$$
\frac{\partial^{2} h}{\partial z^{2}}-\frac{3}{z} \frac{\partial h}{\partial z}-\frac{\partial^{2} h}{\partial \tau^{2}}+\nabla^{2} h=0
$$

where $h=\phi, b, \hat{\Psi}$ and $\hat{E}$. Taking the solutions of the form $h\left(z, \tau, x^{i}\right)=(z / l)^{2} S(z) e^{-i \omega \tau} e^{i \mathbf{k x}}$, the equation for $S(z)$ is obtained as

$$
S(z)^{\prime \prime}+\frac{1}{z} S(z)^{\prime}+\left(m^{2}-\frac{4}{z^{2}}\right) S(z)=0, \quad m^{2}=\omega^{2}-\mathbf{k}^{2}
$$

where $S(z)^{\prime}$ denotes $d S(z) / d z$. The solutions are given by linear combinations of the Bessel function and the Neumann function $J_{2}(m z)+a_{m} N_{2}(m z)$. The coefficient $a_{m}$ is determined by the boundary conditions at $z \rightarrow \infty$. We take the boundary conditions that the positive frequency functions are ingoing at $z \rightarrow \infty$. Then the solution is given by

$$
\begin{gathered}
\hat{h}=\left(\frac{z}{l}\right)^{2} \int \frac{d^{3} \mathbf{k}}{(2 \pi)^{3}} \int d m h_{m}(\mathbf{k}) H_{2}^{(1)}(m z) e^{-i \omega \tau} e^{i \mathbf{k x},} \\
(h=\psi, b, \hat{\Psi}, \hat{E}),
\end{gathered}
$$

where $H_{2}^{(1)}$ is the Hunkel function of the first kind. From the gauge fixing conditions, the coefficients $h_{m}(\mathbf{k})$ satisfy

$$
\begin{aligned}
& \phi_{m}(\mathbf{k})=\frac{2 \mathbf{k}^{4} l^{2}}{2 \mathbf{k}^{2}+3 m^{2}} E_{m}(\mathbf{k}), \\
& b_{m}(\mathbf{k})=-\frac{4 i \sqrt{\mathbf{k}^{2}+m^{2}} \mathbf{k}^{2} l^{2}}{2 \mathbf{k}^{2}+3 m^{2}} E_{m}(\mathbf{k}), \\
& \hat{\Psi}_{m}(\mathbf{k})=-\frac{m^{2} \mathbf{k}^{2} l^{2}}{2 \mathbf{k}^{2}+3 m^{2}} E_{m}(\mathbf{k}), \\
& \hat{E}_{m}(\mathbf{k})=l^{2} E_{m}(\mathbf{k}),
\end{aligned}
$$

where $E_{m}(\mathbf{k})$ is the arbitrary coefficient. This corresponds to the one degree of freedom of the 5D scalar graviton and represents the spectrum of the gravitational waves emitted from the perturbed brane.

(2) Make the coordinate transformation to provide the cosmological background:

$$
z=l(f(u)-g(v)), \quad \tau=l(f(u)+g(v)),
$$

The perturbations in the cosmological background can be obtained using Eq. (8) as 


$$
\begin{aligned}
h= & J e^{-2 \alpha(y, t)} \int \frac{d^{3} \mathbf{k}}{(2 \pi)^{3}} \int d m h_{m}(\mathbf{k}) \\
& \times H_{2}^{(1)}\left(m l e^{-\alpha(y, t)}\right) l^{2} E_{m} e^{-i \omega \tau(t, y)},
\end{aligned}
$$

where $J$ is the factor which comes from the Jacobian of the transformation (27):

$$
\begin{aligned}
& \frac{\partial \tau}{\partial y}=l\left(-f^{\prime}(u)+g^{\prime}(v)\right)=l \dot{\alpha} e^{-\alpha}, \\
& \frac{\partial z}{\partial y}=-l\left(f^{\prime}(u)+g^{\prime}(v)\right)=-l \alpha^{\prime} e^{-\alpha}, \\
& \frac{\partial \tau}{\partial t}=l\left(f^{\prime}(u)+g^{\prime}(v)\right)=l \alpha^{\prime} e^{-\alpha}, \\
& \frac{\partial z}{\partial t}=l\left(f^{\prime}(u)-g^{\prime}(v)\right)=-l \dot{\alpha} e^{-\alpha} .
\end{aligned}
$$

In addition, we make (infinitesimal) coordinate transformations $\bar{x}^{M}=x^{M}+\xi^{M}$ to ensure that the brane is located at $\bar{y}$ $=0$. After imposing the gauge conditions $g_{y \mu}=0$, there remains one freedom of the coordinate transformation $\left(\xi^{y}\right)$. Then we take a slicing along the spacetime $\bar{y}=0$ to cut the spacetime.

(3) Cut the spacetime at $\bar{y}=0$ and glue two copies of the remaining spcaetime along $\bar{y}=0$. From the junction conditions the matter on the brane is determined in terms of $E_{m}(\mathbf{k})$ and $\xi^{y}$.

(4) Finally impose the two conditions on the matter perturbations and determine $E_{m}(\mathbf{k})$ and $\xi^{y}$. We will impose the condition on the anisotropic stress and equation of state of the matter.

\section{EVOLUTION OF PERTURBATIONS AT SUPERHORIZON SCALES}

Following the formalism developed in the previous section, we calculate the evolution of the perturbations. To simplify the calculations, we first consider the long-wave perturbations in the brane world. We shall take the limit

$$
\mathbf{k} \rightarrow 0 \text {. }
$$

We will calculate the evolution for late times where the Hubble scales is sufficiently low

$$
H \ll l^{-1} \text {. }
$$

We will take the assumption that the modes with $m e^{-\alpha_{0}}$ $>l^{-1}$ do not contribute to the perturbations in the brane world. Then we assume

$$
m l e^{-\alpha_{0} \ll 1}
$$

The effect of these modes will be discussed in Sec. V.

\section{A. Calculation of perturbations}

(1) In the $\mathbf{k} \rightarrow 0$ limit, we see only $\hat{E}$ survives in Eq. (26). Then we shall start with

$$
d s^{2}=\left(\frac{l}{z}\right)^{2}\left(d z^{2}-d \tau^{2}+\left(\delta_{i j}+2 \hat{E}_{, i j}\right) d x^{i} d x^{j}\right) .
$$

(2) We make the (large) coordinate transformation (6). Since $\hat{E}$ does not change in this coordinate transformation, the metric is given by

$$
d s^{2}=e^{2 \beta(y, t)}\left(d y^{2}-d t^{2}\right)+e^{2 \alpha(y, t)}\left(\delta_{i j}+2 \hat{E}_{, i j}\right) d x^{i} d x^{j} .
$$

Due to the bending of the brane by matter perturbations, the brane is not located at $y=0$. We perform the (infinitesimal) coordinate transformation

$$
x^{M} \rightarrow x^{M}+\xi^{M}, \quad \xi^{M}=\left(\xi^{y}, \xi^{t}, \xi^{, i}\right) .
$$

After this coordinate transformation, the perturbed metric is given by

$$
\begin{aligned}
d s^{2}= & e^{2 \beta(y, t)}\left((1+2 N) d y^{2}-(1+2 \Phi) d t^{2}+2 A d t d y\right) \\
& +e^{2 \alpha(y, t)}\left(\left[(1-2 \Psi) \delta_{i j}+2 E_{, i j}\right] d x^{i} d x^{j}+2 B_{, i} d x^{i} d t\right. \\
& \left.+2 G_{, i} d x^{i} d y\right),
\end{aligned}
$$

where

$$
\begin{aligned}
& \Phi=\dot{\xi}^{t}+\beta^{\prime} \xi^{y}+\dot{\beta} \xi^{t}, \\
& \Psi=-\dot{\alpha} \xi^{t}-\alpha^{\prime} \xi^{y}, \\
& E=\hat{E}+\xi, \\
& B=\xi-e^{2(\beta-\alpha)} \xi^{t}, \\
& A=\dot{\xi}^{y}-\xi^{t^{\prime}}, \\
& G=e^{2(\beta-\alpha)} \xi^{y}+\xi^{\prime}, \\
& N=\xi^{y^{\prime}}+\beta^{\prime} \xi^{y}+\dot{\beta} \xi^{t} .
\end{aligned}
$$

We will denote the power series expansion near the brane as

$$
\Phi(y, t)=\Phi_{0}(t)+\Phi_{1}(t)|y|+\cdots .
$$

We take the gauge condition $G=A=0$ and $B_{0}=0, E_{0}=0$ (see Appendix B1). This determines $\xi^{t}$ and $\xi$ in terms of $\xi^{y}$ :

$$
\begin{aligned}
\xi^{t} & =\int_{0}^{y} d y \dot{\xi}^{y}+\hat{T}_{0}, \quad \hat{T}_{0}=-e^{2 \alpha_{0}} \hat{E}_{0}, \\
\xi & =-\int_{0}^{y} d y e^{2(\beta-\alpha)} \xi^{y}-\hat{E}_{0} .
\end{aligned}
$$

Then we obtain the metric perturbations induced on the brane 


$$
\begin{aligned}
& \Phi_{0}=\beta_{1} \xi_{0}^{y}+\dot{\hat{T}}_{0}, \\
& \Psi_{0}=-\alpha_{1} \xi_{0}^{y}-\dot{\alpha}_{0} \hat{T}_{0}, \\
& N_{0}=\xi_{1}^{y}+\beta_{1} \xi_{0}^{y},
\end{aligned}
$$

and the first derivative of the metric perturbations

$$
\begin{aligned}
& \Phi_{1}=\ddot{\xi}_{0}^{y}+\beta_{1} \xi_{1}^{y}+\beta_{2} \xi_{0}^{y}+\dot{\beta}_{1} \hat{T}_{0}, \\
& \Psi_{1}=-\alpha_{1} \xi_{1}^{y}-\dot{\alpha}_{0} \dot{\xi}_{0}^{y}-\alpha_{2} \xi_{0}^{y}-\dot{\alpha}_{1} \hat{T}_{0}, \\
& N_{1}=\xi_{2}^{y}+\beta_{1} \xi_{1}^{y}+\beta_{2} \xi_{0}^{y}+\dot{\beta}_{1} \hat{T}_{0}, \\
& B_{1}=e^{-2 \alpha_{0}}\left(-2 \dot{\xi}_{0}^{y}+2 \dot{\alpha}_{0} \xi_{0}^{y}+2\left(\alpha_{1}-\beta_{1}\right) \hat{T}_{0}\right), \\
& E_{1}=\hat{E}_{1}-e^{-2 \alpha_{0}} \xi_{0}^{y} .
\end{aligned}
$$

(3) We take the perturbed energy momentum in the 5D spacetime as

$$
\delta T_{N}^{M}=\left(\begin{array}{ccc}
0 & 0 & 0 \\
0 & -\delta \rho & (\epsilon+p) e^{-\alpha_{0}} v_{, i} \\
0 & -(\epsilon+p) e^{\alpha_{0}} v_{, i} & \delta p \delta_{i j}
\end{array}\right) \delta(y),
$$

where we assume the anisotropic stress of the matter is zero. The jump of the first derivative of the metric perturbations should be equated with the matter perturbations on the brane. This junction condition is obtained as (see Appendix B1)

$$
\begin{aligned}
& \Psi_{1}=-\alpha_{1} N_{0}+\frac{1}{6} \kappa^{2} \delta \rho, \\
& \Phi_{1}=\beta_{1} N_{0}+\kappa^{2}\left(\frac{\delta \rho}{3}+\frac{\delta p}{2}\right), \\
& B_{1}=-2\left(\beta_{1}-\alpha_{1}\right) e^{\alpha_{0}} v, \\
& E_{1}=0 .
\end{aligned}
$$

Combining Eqs. (41) and (43), we can write the perturbed energy momentum tensor in terms of $\xi^{y}$ and $E_{m}(\mathbf{k})$ :

$$
\begin{aligned}
\kappa^{2} \delta \rho= & -6\left(\dot{\alpha}_{0} \dot{\xi}_{0}^{y}-\dot{\alpha}_{0}^{2} \xi_{0}^{y}+\dot{\alpha}_{1} \hat{T}_{0}\right), \\
\kappa^{2} \delta p= & 2\left(\ddot{\xi}_{0}^{y}+2 \dot{\alpha}_{0} \dot{\xi}_{0}^{y}-\left(2 \ddot{\alpha}_{0}+3 \dot{\alpha}_{0}^{2}\right) \xi_{0}^{y}\right. \\
& \left.+\left(\dot{\beta}_{1}+2 \dot{\alpha}_{1}\right) \hat{T}_{0}\right), \\
\kappa^{2}(\rho+p) e^{\alpha_{0}} v= & 2 \dot{\xi}_{0}^{y}-2 \dot{\alpha}_{0} \xi_{0}^{y}+\kappa^{2}(\rho+p) \hat{T}_{0}, \\
0= & \hat{E}_{1}-e^{-2 \alpha_{0}} \xi_{0}^{y} .
\end{aligned}
$$

(4) Imposing the constraints on the matter determines the unknown function $\xi^{y}$ and $E_{m}$. First $\xi_{0}^{y}$ is determined by the shareless condition:

$$
\xi_{0}^{y}=e^{2 \alpha_{0}} \hat{E}_{1} .
$$

The coefficient $E_{m}$ is determined demanding the equation of state $\delta p=c_{s}^{2} \delta \rho$. In the coordinate $(t, y), \hat{E}$ can be written as

$$
\hat{E}(t, y)=e^{-2 \alpha(y, t)} \int d m H_{2}^{(1)}\left(m l e^{-\alpha(y, t)}\right) l^{2} E_{m} e^{-i m \tau(t, y)} .
$$

Let us take the limit $m l e^{-\alpha} \ll 1$. Then we can use the asymptotic form of the Hunkel function for small argument:

$$
H_{2}^{(1)}(z) \sim \frac{2}{z^{2}}+\frac{1}{2}+\mathcal{O}\left(z^{2}, z^{4}, \ldots\right),
$$

where we neglect the overall numerical coefficient. $\hat{E}$ can be evaluated as

$$
\hat{E}(t, y)=\int d m\left(\frac{2}{m^{2} l^{2}}+\frac{1}{2} e^{-2 \alpha(y, t)}\right) l^{2} E_{m} e^{-i m \tau(t, y)},
$$

Using the Jacobian of the transformation (6)

$$
\begin{aligned}
& \left.\frac{\partial \tau}{\partial y}\right|_{y=0}=l\left(-f^{\prime}(t / l)+g^{\prime}(t / l)\right)=l \dot{\alpha}_{0} e^{-\alpha_{0}}, \\
& \left.\frac{\partial z}{\partial y}\right|_{y=0}=-l\left(f^{\prime}(t / l)+g^{\prime}(t / l)\right)=-l \alpha^{\prime} e^{-\alpha_{0}}, \\
& \left.\frac{\partial \tau}{\partial t}\right|_{y=0}=l\left(f^{\prime}(t / l)+g^{\prime}(t / l)\right)=l \alpha_{1} e^{-\alpha_{0}}, \\
& \left.\frac{\partial z}{\partial t}\right|_{y=0}=l\left(f^{\prime}(t / l)-g^{\prime}(t / l)\right)=-l \dot{\alpha}_{0} e^{-\alpha_{0}},
\end{aligned}
$$

we can verify the following equations about $\xi_{0}^{y}=e^{2 \alpha_{0}} \hat{E}_{1}$ and $\hat{T}_{0}=-e^{2 \alpha_{0}} \hat{\dot{E}}_{0}$ :

$$
\begin{aligned}
\alpha_{1} \xi_{0}^{y}=\beta_{1} \xi_{0}^{y}= & \int d m\left(-1+2 i \frac{\dot{\alpha}_{0}}{m e^{-\alpha_{0}}}\right) E_{m} e^{-i m \tau}, \\
\alpha_{1} \dot{\xi}_{0}^{y}= & \int d m\left(-2 \dot{\alpha}_{0}-i m e^{-\alpha_{0}}+2 i \frac{\ddot{\alpha}_{0}+\dot{\alpha}_{0}^{2}}{m e^{-\alpha_{0}}}\right) \\
& \times E_{m} e^{-i m \tau},
\end{aligned}
$$




$$
\begin{aligned}
\alpha_{1} \ddot{\xi}_{0}^{y}= & \int d m\left(-4 \ddot{\alpha}_{0}-2 \dot{\alpha}_{0}^{2}+m^{2} e^{-2 \alpha_{0}}\right. \\
& \left.-i m \dot{\alpha}_{0} e^{-\alpha_{0}}+2 i \frac{\dot{\alpha}_{0}+3 \dot{\alpha}_{0} \ddot{\alpha}_{0}+\dot{\alpha}_{0}^{3}}{m e^{-\alpha_{0}}}\right) \\
& \times E_{m} e^{-i m \tau},
\end{aligned}
$$

and

$$
\begin{aligned}
\dot{\alpha}_{0} \hat{T}_{0} & =\int d m\left(-2 i \frac{\dot{\alpha}_{0}}{m e^{-\alpha_{0}}}\right) E_{m} e^{-i m \tau}, \\
\dot{\hat{T}}_{0} & =\int d m\left(2-2 i \frac{\dot{\alpha}_{0}}{m e^{-\alpha_{0}}}\right) E_{m} e^{-i m \tau},
\end{aligned}
$$

where we used $\dot{\alpha}_{0}^{2} l^{2}, \ddot{\alpha}_{0} l^{2} \sim(H l)^{2} \ll 1$. Then, we obtain

$$
\begin{aligned}
\kappa^{2} \alpha_{1} \delta \rho= & \int d m\left(6 \dot{\alpha}_{0}^{2}+6 i m \dot{\alpha}_{0} e^{-\alpha_{0}}\right) E_{m} e^{-i m \tau}, \\
\kappa^{2} \alpha_{1} \delta p= & \int d m\left(6 w \dot{\alpha}_{0}^{2}-6 i m \dot{\alpha}_{0} e^{-\alpha_{0}}\right. \\
& \left.+2 m^{2} e^{-2 \alpha_{0}}\right) E_{m} e^{-i m \tau},
\end{aligned}
$$

where $p=w \rho$. We assume $c_{s}^{2}=w=$ const. Then we observe that the equation of state $\delta p=w \delta \rho$ is satisfied if $m e^{-\alpha_{0}}$ $\ll H$. It implies that $E_{m}$ should select the modes with $m e^{-\alpha_{0} \ll H}$. Note that the assumption $m l e^{-\alpha_{0} \ll 1}$ is consistent with the result that only the modes with $m e^{-\alpha_{0}<H}$ contribute to the perturbations for late times $H<l^{-1}$.

\section{B. Evolution of perturbations at superhorizon scales}

Let us evaluate the metric perturbations $\Phi_{0}$ and $\Psi_{0}$ in the brane world (40). From Eqs. (40), (50), and (51), we obtain

$$
\Phi_{0}=\Psi_{0}=\int d m E_{m} e^{-i m \tau}
$$

For late times where the Hubble horizon is sufficiently larger than the curvature scale of the AdS spacetime $H<l^{-1}, \tau(t)$ is given by (see Appendix A)

$$
\tau(t) \sim l e^{-\alpha_{0}}\left(\begin{array}{l}
t \\
l
\end{array}\right)^{1-2 / 3(1+w)}
$$

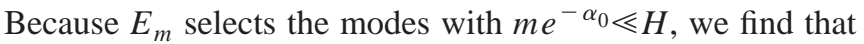
$m \tau \ll 1$. Then the metric fluctuations are constant. The density fluctuations becomes

$$
4 \pi G_{4} \delta \rho=-3 \dot{\alpha}_{0}^{2} \Phi_{0},
$$

where we use $\kappa^{4} \sigma=48 \pi G_{4}$. We finally obtain the metric perturbations and density fluctuations on the brane

$$
\frac{\delta \rho}{\rho}=-2 \Phi_{0}=\text { const. }
$$

This is one of the main results of our work. We notice that the solutions for metric perturbations and density perturbations are identical with those obtained in the conventional 4D cosmology. Note that, if $w \neq c_{s}^{2}$, the modes with $m e^{-\alpha_{0}}$ $>H$ should contribute to $\Phi_{0}$ in order to satisfy $\delta p=c_{s}^{2} \delta \rho$. Once these modes contribute to $\Phi_{0}, \Phi_{0}$ is no longer constant. This also agrees with the 4D cosmology.

\section{EVOLUTION OF PERTURBATIONS AT SUBHORIZON SCALES}

In this section we shall study the evolution of perturbations at subhorizon scales $\mathbf{k} e^{-\alpha_{0}}>H$. We derive the equation to determine $E_{m}(\mathbf{k})$ for each mode with $\mathbf{k}$. We again use the assumption that the modes with $m e^{-\alpha_{0}}>l^{-1}$ do not contribute to the perturbations in the brane world. Then we investigate the late time evolution of perturbations at subhorizon scales larger than the AdS curvature scale $l\left(l^{-1}>\mathbf{k} e^{-\alpha_{0}}\right.$ $>H)$.

\section{A. Calculations of perturbations}

(1) We start with the perturbed AdS spacetime

$$
\begin{aligned}
d s^{2}= & \left(\frac{l}{z}\right)^{2}\left(d z^{2}-(1+2 \phi) d \tau^{2}+2 b_{, i} d x^{i} d \tau\right. \\
& \left.+\left[(1-2 \hat{\Psi}) \delta_{i j}+2 \hat{E}_{, i j}\right] d x^{i} d x^{j}\right),
\end{aligned}
$$

where $\phi, b, \hat{\Psi}$ and $\hat{E}$ is given by Eq. (26).

(2) The perturbations in $\left(y, t, x^{i}\right)$ coordinate is obtained by the coordinate transformation. The resulting metric becomes [see Eqs. (28) and (29)]

$$
\begin{aligned}
d s^{2}= & e^{2 \beta(y, t)}\left((1+2 \hat{N}) d y^{2}-(1+2 \hat{\Phi}) d t^{2}+2 \hat{A} d t d y\right) \\
& +e^{2 \alpha(y, t)}\left(\left[(1-2 \hat{\Psi}) \delta_{i j}+2 \hat{E}_{, i j}\right] d x^{i} d x^{j}+2 \hat{B}_{, i} d x^{i} d t\right. \\
& \left.+2 \hat{G}_{, i} d x^{i} d y\right)
\end{aligned}
$$

where

$$
\begin{aligned}
& \hat{\Phi}=\left(l \alpha^{\prime}\right)^{2} e^{-2 \beta} \phi, \\
& \hat{B}=\left(l \alpha^{\prime}\right) e^{-\alpha} b, \\
& \hat{N}=-(l \dot{\alpha})^{2} e^{-2 \beta} \phi, \\
& \hat{A}=-2\left(l^{2} \dot{\alpha} \alpha^{\prime}\right) e^{-2 \beta} \phi, \\
& \hat{G}=(l \dot{\alpha}) e^{-\alpha} b .
\end{aligned}
$$

In addition, we perform the (infinitesimal) coordinate transformation by

$$
x^{M} \rightarrow x^{M}+\xi^{M}, \quad \xi^{M}=\left(\xi^{y}, \xi^{0}, \xi^{, i}\right) .
$$


We will take the gauge condition $G=A=0$ and $B_{0}=0, E_{0}$ $=0$. This determines $\xi^{t}$ and $\xi$ in terms of $\xi^{y}$ as

$$
\begin{aligned}
& \xi^{t}=\int_{0}^{y} d y\left(\hat{A}+\dot{\xi}^{y}\right)+\hat{T}_{0}, \quad \hat{T}_{0}=e^{2 \alpha_{0}}\left(\hat{B}_{0}-\dot{\hat{E}}\right), \\
& \xi=-\int_{0}^{y} d y\left(\hat{G}+e^{2(\beta-\alpha)} \xi^{y}\right)-\bar{E}_{0} .
\end{aligned}
$$

Then we obtain the metric perturbations on the brane

$$
\begin{aligned}
& \Phi_{0}=\hat{\Phi}+\beta_{1} \xi_{0}^{y}+\dot{\hat{T}}_{0}, \\
& \Psi_{0}=\hat{\Psi}-\alpha_{1} \xi_{0}^{y}-\dot{\alpha}_{0} \hat{T}_{0}, \\
& N_{0}=\hat{N}_{0}+\xi_{1}^{y}+\beta_{1} \xi_{0}^{y},
\end{aligned}
$$

and the first derivative of the metric perturbations

$$
\begin{aligned}
& \Phi_{1}=\ddot{\xi}_{0}^{y}+\beta_{1} \xi_{1}^{y}+\beta_{2} \xi_{0}^{y}+\hat{\Phi}_{1}+\dot{\hat{A}}_{0}+\dot{\beta}_{1} \hat{T}_{0}, \\
& \Psi_{1}=-\alpha_{1} \xi_{1}^{y}-\dot{\alpha}_{0} \dot{\xi}_{0}^{y}-\alpha_{2} \xi_{0}^{y}+\hat{\Psi}_{1}-\dot{\alpha}_{0} \hat{A}_{0}-\dot{\alpha}_{1} \hat{T}_{0}, \\
& N_{1}=\xi_{2}^{y}+\beta_{1} \xi_{1}^{y}+\beta_{2} \xi_{0}^{y}+\hat{N}_{1}+\dot{\beta}_{1} \hat{T}_{0}, \\
& B_{1}=e^{-2 \alpha_{0}}\left(-2 \dot{\xi}_{0}^{y}+2 \dot{\alpha}_{0} \xi_{0}^{y}-2 \beta_{1} \hat{T}_{0}-2 \alpha_{1} e^{2 \alpha_{0}} \dot{\hat{E}}_{0}\right. \\
& \left.-\hat{A}_{0}+e^{2 \alpha_{0}} \hat{B}_{1}-e^{2 \alpha_{0}} \dot{\hat{G}}_{0}\right), \\
& E_{1}=\hat{E}_{1}-e^{-2 \alpha_{0}} \xi_{0}^{y}-\hat{G}_{0} .
\end{aligned}
$$

(3) Combining the junction conditions (43) and (63), we can write matter in terms of $\xi^{y}$ and $E_{m}(\mathbf{k})$ :

$$
\begin{aligned}
\kappa^{2} \delta \rho= & -6\left(\dot{\alpha}_{0} \dot{\xi}_{0}^{y}+\left(\alpha_{2}-\alpha_{1} \beta_{1}\right) \xi_{0}^{y}-\hat{\Psi}_{1}\right. \\
& \left.+\dot{\alpha}_{0} \hat{A}_{0}+\dot{\alpha}_{1} \hat{T}_{0}-\alpha_{1} \hat{N}_{0}\right), \\
\kappa^{2} \delta p= & 2\left(\ddot{\xi}_{0}^{y}+2 \dot{\alpha}_{0} \dot{\xi}_{0}^{y}+\left(2 \alpha_{2}+\beta_{2}-\beta_{1}^{2}\right.\right. \\
& \left.-2 \alpha_{1} \beta_{1}\right) \xi_{0}^{y}+\hat{\Phi}_{1}-2 \hat{\Psi}_{1}+\dot{\hat{A}}_{0}+2 \dot{\alpha}_{0} \hat{A}_{0} \\
& \left.+\left(\dot{\beta}_{1}+2 \dot{\alpha}_{1}\right) \hat{T}_{0}-\left(\beta_{1}+2 \alpha_{1}\right) \hat{N}_{0}\right), \\
\kappa^{2}(\rho+P) e^{\alpha_{0} v}= & 2 \dot{\xi}_{0}^{y}-2 \dot{\alpha}_{0} \xi_{0}^{y}-e^{2 \alpha_{0}} \hat{B}_{1}+2 \alpha_{1} e^{2 \alpha_{0}} \dot{\hat{E}}_{0} \\
& +2 \beta_{1} \hat{T}_{0}+e^{2 \alpha_{0}} \dot{\hat{G}}_{0}+\hat{A}_{0}, \\
0= & -2 e^{-2 \alpha_{0}} \xi_{0}^{y}+2 \hat{E}_{1}-2 \hat{G}_{0} .
\end{aligned}
$$

(4) Imposing the condition on the anisotoropic stress $\pi_{T}$ and the equation of state $c_{s}^{2}=\delta p / \delta \rho$ determines $\xi_{0}^{y}$ and $E_{m}(\mathbf{k})$. Substituting $\xi_{0}^{y}=e^{2 \alpha_{0}}\left(\hat{E}_{1}-\hat{G}_{0}\right)$ into $\delta p-c_{s}^{2} \delta \rho=0$, we obtain the following form of the equation for each $\mathbf{k}$ :

$$
\int d m F(t, m ; \mathbf{k}) E_{m}(\mathbf{k})=0 .
$$

Because the detailed form of $F(t, m ; \mathbf{k})$ is rather complicated, we omit it here. Defining the Fourier transformation of the function $F(t, m ; \mathbf{k})$ by

$$
F(t, m ; \mathbf{k})=\int d m^{\prime} \widetilde{F}\left(m^{\prime}, m ; \mathbf{k}\right) e^{-i m t},
$$

this equation becomes

$$
\int d m \widetilde{F}\left(m^{\prime}, m ; \mathbf{k}\right) E_{m}(\mathbf{k})=0 .
$$

The problem is to find the eigenstate of the matrix $F\left(m^{\prime}, m\right)$ with eigenvalue 0 . Since $F(t, m ; \mathbf{k})$ consists of the combination of the oscillating function, the existence of the solution is very likely.

\section{B. Evolution of perturbations at subhorizon scales}

The equation to determine $E_{m}(\mathbf{k})$ is rather complicated. However we can deduce the evolution of perturbations for late times $H<l^{-1}$ from the following arguments. We take the assumption that the massive modes with $m e^{-\alpha_{0}}>l^{-1}$ can be neglected. We can evaluate the metric perturbations as

$$
\begin{aligned}
& \Phi_{0}=\int d m \frac{3 m^{2}}{2 \mathbf{k}^{2}+3 m^{2}}\left(1+\frac{1}{6}\left(\mathbf{k} l e^{-\alpha_{0}}\right)^{2}\right) E_{m}(\mathbf{k}) e^{-i \omega \tau}, \\
& \Psi_{0}=\int d m \frac{3 m^{2}}{2 \mathbf{k}^{2}+3 m^{2}}\left(1-\frac{1}{6}\left(\mathbf{k} l e^{-\alpha_{0}}\right)^{2}\right) E_{m}(\mathbf{k}) e^{-i \omega \tau},
\end{aligned}
$$

where we neglect the terms of the order $\mathcal{O}\left(\left(m l e^{-\alpha_{0}}\right)^{2}\right)$. Then we find that

$$
\Phi_{0}-\Psi_{0}=\mathcal{O}\left(\left(\mathbf{k} l e^{-\alpha_{0}}\right)^{2}\right) .
$$

For $\mathbf{k} l e^{-\alpha_{0}} \rightarrow 0$ but not necessarily $\mathbf{k} e^{-\alpha_{0}}<H$, these agree with the result obtained in Eq. (53). A notable point is that this equation is sufficient to show that the $4 \mathrm{D}$ cosmology is reproduced. From the 5D Einstein equation, we have already obtained the three equations for $\Phi_{0}, \Psi_{0}, \delta \rho$ and $v$ [conservation of the energy momentum (17) and trace-part of the Einstein equation (18)]. Thus, for $\mathbf{k} l e^{-\alpha_{0} \ll 1}$, we have closed set of equations about the metric perturbations and density fluctuations, which is identical with the one obtained in the conventional $4 \mathrm{D}$ equation. Then we conclude that if the effect of the massive modes with $m l e^{-\alpha_{0}}>1$ can be neglected, the cosmological perturbations are not interfered by the extra dimension. Whether the modes with $m l e^{-\alpha_{0}}$ $>1$ contribute to the perturbations or not is determined by $E_{m}(\mathbf{k})$ which can be obtained from Eq. (67).

\section{DEVIATION FROM 4D COSMOLOGY}

In the previous two sections, we show that the late time evolution of the perturbations is not modified by the $5 \mathrm{D}$ graviton if the effect of the modes with $m e^{-\alpha_{0} \gg l^{-1}}$ is negligible. Let us investigate the effect of these massive modes. 

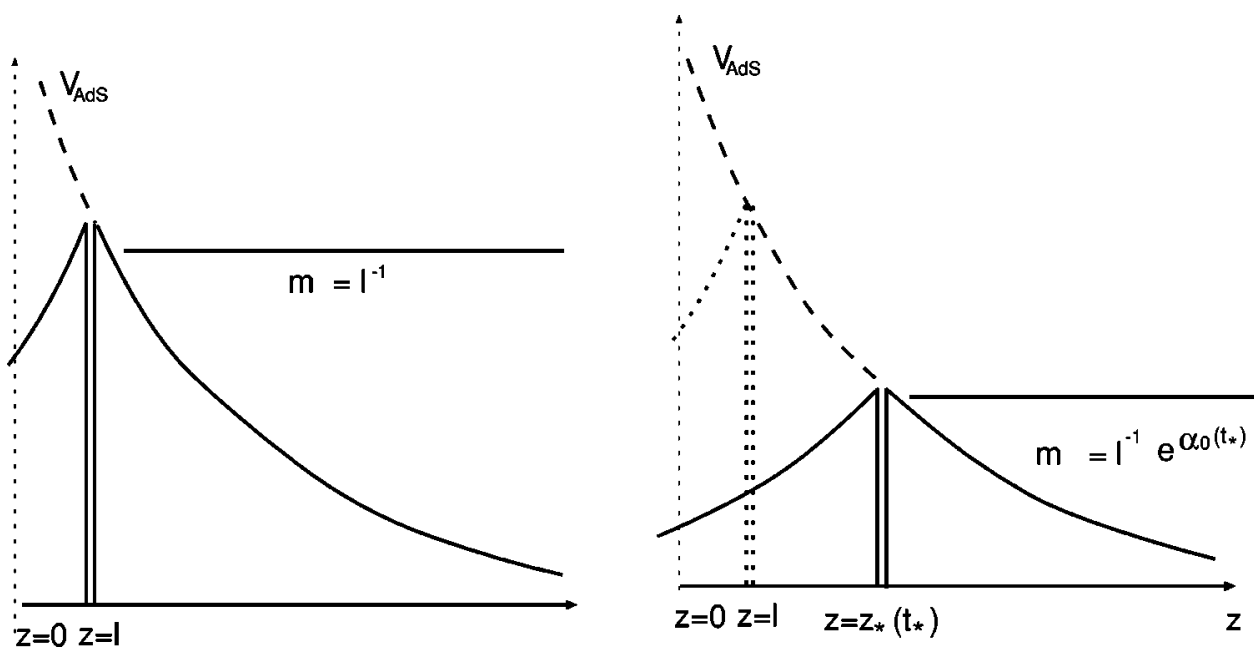

FIG. 4. Volcano potential for $5 \mathrm{D}$ gravity. The location of the brane determines the height of the "barrier" which protects the brane from massive modes.

To simplify the calculation, we assume $\mathbf{k} \rightarrow 0$. Without taking the limit $m l e^{-\alpha_{0} \ll 1,} \alpha_{1} \xi_{0}^{y}$ and $\dot{\alpha}_{0} \hat{T}$ becomes

$$
\begin{aligned}
& \alpha_{1} \xi_{0}^{y}=-\int d m\left(m l e^{-\alpha_{0}}\right) E_{m} H_{1}^{(1)}\left(m l e^{-\alpha_{0}}\right) e^{-i m \tau}
\end{aligned}
$$

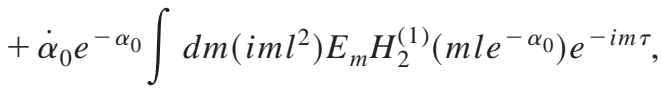

$$
\begin{aligned}
& \dot{\alpha}_{0} \hat{T}_{0}=\dot{\alpha}_{0}^{2} l^{2} \int d m\left(m l e^{-\alpha_{0}}\right) E_{m} H_{1}^{(1)}\left(m l e^{-\alpha_{0}}\right) e^{-i m \tau} \\
& -\dot{\alpha}_{0} e^{-\alpha_{0}} \int d m\left(i m l^{2}\right) E_{m} H_{2}^{(1)}\left(m l e^{-\alpha_{0}}\right) e^{-i m \tau} .
\end{aligned}
$$

Then we can evaluate $\Psi_{0}$ as

$$
\begin{aligned}
\Psi_{0} & =\left(1-\dot{\alpha}_{0}^{2} l^{2}\right) \int d m \Psi_{m}(t) E_{m}(\mathbf{k}) e^{-i \omega \tau}, \\
\Psi_{m}(t) & =\left(m l e^{-\alpha_{0}}\right) H_{1}^{(1)}\left(m l e^{-\alpha_{0}}\right) .
\end{aligned}
$$

We shall investigate the effect of the massive modes. The evolution of $\Psi_{m}$ can be estimated using the asymptotic form of the Hunkel function for large argument $H_{1}^{(1)}(z) \propto 1 / \sqrt{z}$. We find that $\Psi_{m}(t)$ behaves as

$$
\Psi_{m}(t) \propto e^{-\alpha_{0}(t) / 2} \quad\left(m>l^{-1} e^{\alpha_{0}}\right) .
$$

Then, the modes with

$$
m>m_{e f f}=l_{e f f}^{-1}, \quad l_{e f f}=l e^{-\alpha_{0}},
$$

modify the evolution of the metric perturbations and hence of the density fluctuations. Because we have normalized the scale factor as $e^{\alpha_{0}\left(t=t_{\text {present }}\right)}=1, l_{\text {eff }}$ becomes larger than $l$ for early times.

The result can be interpreted as follows. For late times, the trajectory of the brane is identical with the RS brane (see Fig. 2). Thus the behavior of the gravity in the brane world for late times can be deduced from the arguments for RS solution. In the coordinate system $\left(z, \tau, x^{i}\right)$, the perturbations $h\left(z, \tau, x^{i}\right)$ satisfies the wave equation (23). Defining $h\left(z, \tau, x^{i}\right)=(z / l)^{3 / 2} \psi(z) e^{-i \omega \tau} e^{i \mathbf{k x}}$, the wave equation can be rewritten as

$$
\left(-\frac{1}{2} \frac{d^{2}}{d z^{2}}+V_{A d S}(z)\right) \psi(z)=\frac{1}{2} m^{2} \psi(z), \quad V_{A d S}(z)=\frac{15}{8} \frac{1}{z^{2}} .
$$

The problem can be understood as the potential problem in one dimensional quantum mechanics where $m$ represents the energy. If we cut the AdS spacetime at $z=l$ and put the brane there, the potential term proportional to $\delta(z-l)$ appears. Imposing the $Z_{2}$ symmetry across the brane, the potential becomes a "volcano" potential. The term proportional to the delta function is responsible for the normalizable zero mode of the 5D graviton which reproduces the 4D gravity on the brane. The brane is protected from the the massive modes by the potential barrier $V_{A d S}$ (see Fig. 4). Only the modes with large $m$ can affect the gravity on the brane. Let us consider the cosmological brane for early times. For early times, the brane is located at large $z>l$. For larger $z$, the potential barrier $V_{A d S}$ is lower, then the mode with smaller $m$ can affect the gravity on the brane. This picture is consistent with the result that the modes with large $m>m_{e f f}=l^{-1} e^{\alpha_{0}}$ can modify the evolution of the metric perturbations because $m_{\text {eff }}$ becomes smaller for early times.

\section{DISCUSSIONS}

The cosmological perturbations in the brane world provide useful tests for the brane world idea. This is because the perturbations in the brane world interact with the perturbations in the bulk which is inherent nature of perturbations in the brane world. The dynamics of the brane cannot be separated from the dynamics of the bulk. This is because the inhomogeneous fluctuations on the brane inevitably produces the gravitational waves in the bulk, which in turn affect the evolution of perturbations on the brane. Thus, naively, we think the evolution of the cosmological perturbations is modified significantly.

We showed that this is not a case for late time $H<l^{-1}$ 
and at scales larger than the AdS curvature scale. The metric perturbations become frozen once the perturbations exit the horizon as in the conventional $4 \mathrm{D}$ cosmology. This result is important for the inflationary scenario in the brane world. If the scale $l^{-1}$ is sufficiently higher than the scales of the inflation, and if heavy graviton modes may be neglected the constancy of the curvature perturbations can be used to estimate the scalar temperature anisotropies of the CMB at large scales. Our results are consistent with those of Ref. [24], where curvature perturbations on large scales is shown to be conserved, and the density perturbations generated during high-energy inflation on the brane are calculated.

The assumption in obtaining the above results is that the effect of massive gravitons with $m>m_{e f f}=e^{\alpha_{0}} l^{-1}$ can be neglected. The contribution of these modes depends on the initial spectrum of the fluctuations. If the primordial fluctuations are generated during inflation at low energies $H<l^{-1}$, heavy gravitions are significantly suppressed [26]. Then, in this case, the assumption seems to be natural.

Another modification of the evolution arises if the scales of the perturbations becomes smaller than $l$. This modification is not important for late time evolution because the cosmological scales is significantly larger than $l$. However at the beginning of the inflation, we should consider the scales comparable to $l$. Then the modification becomes important to predict the primordial spectrum of the fluctuations during inflation.

The key to quantify these modifications is the understanding of $E_{m}(\mathbf{k})$. The equation for $E_{m}(\mathbf{k})$ obtained in this paper might give a way to estimate the effect of the interaction with bulk graviton on the perturbations in the brane world, which is the intrinsic feature of the brane world cosmology.

Finally, we comment on the possibility for the extension of the present work. In this paper, we have considered the spatially flat universe. The extension to the nonflat universe may be possible using the coordinate system in Ref. [14]. The extension of our method to vector and tensor perturbations is straightforward. The tensor perturbations were calculated in the de Sitter background and the agreement with 4D gravity was demonstrated in a certain limit [25]. It will be interesting to investigate the effect of the vector components of 5D graviton on the 4D cosmological perturbations.

Note added. While the present work was being completed, related papers [27-30] appeared on the hep-th.

\section{ACKNOWLEDGMENTS}

We would like to thank S. Kawai, T. Shiromizu, and T. Tanaka for helpful comments. The work of K.K. was supported by the JSPS.

\section{APPENDIX A: BACKGROUND—EINSTEIN EQUATION AND JUNCTION CONDITION}

In this appendix, we derive the junction conditions and obtain a solution for the background. The junction conditions can be obtained from the 5D Einstein equation:

$$
\begin{aligned}
G_{N}^{M} & =\frac{6}{l^{2}} \delta_{N}^{M}+\kappa^{2} \frac{\sqrt{-g_{\text {brane }}}}{\sqrt{-g}} T_{N}^{M} \\
& =\frac{6}{l^{2}} \delta_{N}^{M}+e^{-\beta} \kappa^{2} T_{N}^{M} \quad\left(M, N=y, t, x^{i}\right) .
\end{aligned}
$$

We take for the energy momentum tensor in the 5D spacetime

$$
T_{N}^{M}=[-\sigma \operatorname{diag}(0,1,1,1,1)+\operatorname{diag}(0,-\rho, p, p, p)] \delta(y) .
$$

The jump of the first derivative of $\alpha(y, t)$ and $\beta(y, t)$ gives the $\delta(y)$ function to the Einstein tensor. The Einstein tensor is given by

$$
\begin{aligned}
& G_{0}^{0}=-3 e^{-2 \beta}\left(\dot{\alpha}^{2}+\dot{\alpha} \dot{\beta}-\alpha^{\prime \prime}-2 \alpha^{\prime 2}+\alpha^{\prime} \beta^{\prime}\right), \\
& G_{y}^{y}=3 e^{-2 \beta}\left(-\ddot{\alpha}-2 \dot{\alpha}^{2}+\dot{\alpha} \dot{\beta}+\alpha^{\prime 2}+\alpha^{\prime} \beta^{\prime}\right), \\
& G_{y}^{0}=-3 e^{-2 \beta}\left(\beta^{\prime} \dot{\alpha}+\alpha^{\prime} \dot{\beta}-\dot{\alpha}^{\prime}-\dot{\alpha} \alpha^{\prime}\right), \\
& G_{j}^{i}=\delta_{j}^{i} e^{-2 \beta}\left(-2 \ddot{\alpha}-3 \dot{\alpha}^{2}-\ddot{\beta}+2 \alpha^{\prime \prime}+3 \alpha^{\prime 2}+\beta^{\prime \prime}\right) .
\end{aligned}
$$

Equating the coefficient of $\delta(y)$ we obtain the junction conditions

$$
\begin{aligned}
& \alpha_{1}(t)=-\kappa^{2} e^{\beta_{0}}\left(\frac{\sigma}{6}+\frac{\rho(t)}{6}\right), \\
& \beta_{1}(t)=-\kappa^{2} e^{\beta_{0}}\left(\frac{\sigma}{6}-\frac{\rho(t)}{3}-\frac{p(t)}{2}\right) .
\end{aligned}
$$

Following Ref. [9], we make power series expansion of the Einstein equation near the brane. The $y^{0}$ order of the $(y, y)$, $(y, 0)$ component of the Einstein equation gives

$$
\begin{aligned}
& \ddot{\alpha}_{0}+2 \dot{\alpha}_{0}^{2}=4 \pi G_{4}\left(\frac{\rho}{3}-p\right)+\frac{\kappa^{4} \rho(\rho+3 p)}{36}, \\
& \dot{\rho}+3 \dot{\alpha}_{0}(\rho+p)=0
\end{aligned}
$$

where $\kappa^{4} \sigma=48 \pi G_{4}$. The integration of the first equation gives

$$
\dot{\alpha}_{0}^{2}=\frac{8 \pi G_{4}}{3} \rho+\frac{\kappa^{4} \rho^{2}}{36}+e^{-4 \alpha_{0}} C,
$$

where $C$ is the constant of the integration and is proportional to the mass of the AdS-Schwarzshild mass. Because the bulk is AdS spacetime in our solution, $C=0$. These equations are equivalent with Eq. (16). The $y^{0}$ order of $(0,0)$ and $(i, j)$ components of the Einstein equation give $\alpha_{2}$ and $\beta_{2}$ in terms of $\alpha_{0}, \alpha_{1}, \beta_{0}$ and $\beta_{1}$ : 


$$
\begin{aligned}
& \alpha_{2}=\dot{\alpha}_{0}^{2}+\dot{\alpha}_{0} \dot{\beta}_{0}-2 \alpha_{1}^{2}+\alpha_{1} \beta_{1}+\frac{2}{l^{2}}, \\
& \beta_{2}=\dot{\alpha}_{0}^{2}+2 \ddot{\alpha}_{0}+\ddot{\beta}_{0}+\alpha_{1}^{2}-2 \alpha_{1} \beta_{1}+\frac{2}{l^{2}} .
\end{aligned}
$$

Let us find the function $f(u)$ and $g(v)$ for late times. For late times, we can neglect $\mathcal{O}\left((\rho / \sigma)^{2}\right)$. Then the solution for $e^{-\alpha_{0}}$ is given by

$$
e^{-\alpha_{0}}=f(t / l)-g(t / l)=a_{*}\left(\begin{array}{l}
t \\
\bar{l}
\end{array}\right)^{-2 / 3(1+w)} .
$$

Combining this equation with $e^{2 \beta_{0}}=1$,

$$
4 \frac{f^{\prime}(t / l) g^{\prime}(t / l)}{f(t / l)-g(t / l)}=1
$$

we can obtain the first order differential equation for $f(t / l)$,

$$
\begin{aligned}
\dot{f}(t / l)= & \frac{a_{*}}{3(1+w)}\left(\frac{t}{l}\right)^{-(5+3 w) / 3(1+w)} \\
& \times\left(-1+\sqrt{1+\left(\frac{3}{2}(1+w) \frac{t}{l}\right)^{2}}\right) .
\end{aligned}
$$

For late times $t / l \gg 1$, we obtain the solution for $f(t)$ and $g(t)$ :

$$
\begin{aligned}
& f(t / l)=a_{*} \frac{1}{2}\left(\frac{t}{l}\right)^{-2 / 3(1+w)}\left(1+\frac{3(1+w)}{1+3 w} \frac{t}{l}\right)+b_{*}, \\
& g(t / l)=a_{*} \frac{1}{2}\left(\frac{t}{l}\right)^{-2 / 3(1+w)}\left(-1+\frac{3(1+w)}{1+3 w} \frac{t}{l}\right)+b_{*},
\end{aligned}
$$

where $a_{*}$ and $b_{*}$ is the constants of integration. Then we obtain

$$
\tau(t) \sim l e^{-\alpha_{0}}\left(\begin{array}{c}
t \\
l
\end{array}\right)^{1-2 / 3(1+w)}
$$

where we take $b_{*}=0$.

\section{APPENDIX B: PERTURBATIONS-EINSTEIN EQUATION AND JUNCTION CONDITIONS}

\section{Gauge fixing and Einstein tensor}

In this section, we derive the $5 \mathrm{D}$ Einstein equation for perturbed AdS spacetime and derive the junctions conditions. We will concentrate on the scalar perturbations. We put the perturbed 5D AdS spacetime as

$$
\begin{aligned}
d s^{2}= & e^{2 \beta(y, t)}\left((1+2 N) d y^{2}-(1+2 \Phi) d t^{2}+2 A d t d y\right) \\
& +e^{2 \alpha(y, t)}\left(\left[(1-2 \Psi) \delta_{i j}+2 E_{, i j}\right] d x^{i} d x^{j}+2 B_{, i} d x^{i} d t\right. \\
& \left.+2 G_{, i} d x^{i} d y\right) .
\end{aligned}
$$

There are three degrees of freedom in the gauge transformations

$$
x^{M} \rightarrow x^{M}+\xi^{M}, \quad \xi^{M}=\left(\xi^{y}, \xi^{0}, \xi^{, i}\right) .
$$

By this gauge transformations, metric perturbations are transformed as

$$
\begin{aligned}
& \Phi=\hat{\Phi}+\dot{\xi}^{t}+\beta^{\prime} \xi^{y}+\dot{\beta} \xi^{t}, \\
& \Psi=\hat{\Psi}-\dot{\alpha} \xi^{t}-\alpha^{\prime} \xi^{y},
\end{aligned}
$$

$$
E=\hat{E}+\xi
$$

$$
B=\hat{B}+\dot{\xi}-e^{2(\beta-\alpha)} \xi^{t}
$$$$
A=\hat{A}+\dot{\xi}^{y}-\xi^{t^{\prime}}
$$$$
G=\hat{G}+e^{2(\beta-\alpha)} \xi^{y}+\xi^{\prime},
$$

$$
N=\hat{N}+\xi^{y^{\prime}}+\dot{\beta} \xi^{t}+\beta^{\prime} \xi^{y}
$$

Using these degrees of the freedom, we impose the gauge conditions so that the resulting coordinate becomes Gaussian Normal (GN) coordinate because the metric perturbations in the GN coordinate on the brane are the metric perturbations observed by the observers confined to the brane. In the GN coordinate, the transverse component of the metric $g_{y \mu},(\mu$ $\left.=t, x^{i}\right)$ vanish $\left[G\left(y, t, x^{i}\right)=A\left(y, t, x^{i}\right)=0\right]$ and the brane is located at $y=0$. The former conditions are achieved by $\xi$ and $\xi^{t}$ and the latter condition is achieved by $\xi^{y}$. The conditions $G=A=0$ determine $\xi^{t}$ and $\xi$ as

$$
\begin{aligned}
& \xi^{t}=\int_{0}^{y} d y\left(\hat{A}+\xi^{y}\right)+\epsilon^{t}\left(t, x^{i}\right), \\
& \xi=-\int_{0}^{y} d y\left(\hat{G}+e^{2(\beta-\alpha)} \xi^{y}\right)+\epsilon\left(t, x^{i}\right),
\end{aligned}
$$

where $\epsilon^{t}$ and $\epsilon$ are functions with no $y$ dependence. These residual gauge transformations enable us to impose two additional gauge fixing conditions. We take $B_{0}\left(y=0, t, x^{i}\right)$ $=E_{0}\left(y=0, t, x^{i}\right)=0$ gauge on the analogy of the longitudinal gauge in the conventional 4D cosmological perturbations theory. The Einstein tensor is calculated as 


$$
\begin{aligned}
\delta G_{0}^{0}= & e^{-2 \beta}\left(\left(6 \dot{\alpha}^{2}+6 \dot{\alpha} \dot{\beta}\right) \Phi-3 \alpha^{\prime} N^{\prime}-3 \dot{\alpha} \dot{N}-\left(6 \alpha^{\prime \prime}+12 \alpha^{\prime 2}-6 \alpha^{\prime} \beta^{\prime}\right) N-3 \Psi^{\prime \prime}+3(\dot{\beta}+2 \dot{\alpha}) \dot{\Psi}+3\left(\beta^{\prime}-4 \alpha^{\prime}\right) \Psi^{\prime}\right) \\
& +\nabla^{2}\left(e^{-2 \beta}\left[E^{\prime \prime}-(\dot{\beta}+2 \dot{\alpha}) \dot{E}-\left(\beta^{\prime}-4 \alpha^{\prime}\right) E^{\prime}\right]-2 e^{-2 \alpha} \Psi+e^{-2 \alpha} N+e^{-2(\alpha+\beta)}(\dot{\beta}+2 \dot{\alpha}) B\right), \\
\delta G_{y}^{y}= & e^{-2 \beta}\left(-\left(6 \alpha^{\prime 2}+6 \alpha^{\prime} \beta^{\prime}\right) N+3 \alpha^{\prime} \Phi^{\prime}+3 \dot{\alpha} \dot{\Phi}+\left(6 \ddot{\alpha}+12 \dot{\alpha}^{2}-6 \dot{\alpha} \dot{\beta}\right) \Phi+3 \ddot{\Psi}-3(\dot{\beta}-4 \dot{\alpha}) \dot{\Psi}-3\left(\beta^{\prime}+2 \alpha^{\prime}\right) \Psi^{\prime}\right) \\
& +\nabla^{2}\left(e^{-2 \beta}\left[-\ddot{E}+(\dot{\beta}-4 \dot{\alpha}) \dot{E}+\left(\beta^{\prime}+2 \alpha^{\prime}\right) E^{\prime}\right]-2 e^{-2 \alpha} \Psi+e^{-2 \alpha} \Phi+e^{-2(\alpha+\beta)}[\dot{B}+(2 \dot{\alpha}-\dot{\beta}) B]\right), \\
\delta G_{0}^{y}= & e^{-2 \beta}\left(3 \dot{\Psi}^{\prime}+3 \dot{\alpha} \Phi^{\prime}+3 \alpha^{\prime} \dot{N}-3\left(\beta^{\prime}-\alpha^{\prime}\right) \dot{\Psi}-3(\dot{\beta}-\dot{\alpha}) \Psi^{\prime}\right) \\
& +e^{-2 \beta} \nabla^{2}\left(-\dot{E}^{\prime}+\left(\beta^{\prime}-\alpha^{\prime}\right) \dot{E}+(\dot{\beta}-\dot{\alpha}) E^{\prime}+e^{-2 \alpha}\left(-\beta^{\prime} B+\frac{1}{2} B^{\prime}\right)\right), \\
\delta G_{i}^{y}= & e^{-2 \beta}\left(\left(2 \alpha^{\prime}+\beta^{\prime}\right) N+\left(\alpha^{\prime}-\beta^{\prime}\right) \Phi-\Phi^{\prime}+2 \Psi^{\prime}+e^{-2 \beta}\left[\left(3 \dot{\alpha} \alpha^{\prime}+\dot{\alpha}^{\prime}-2 \alpha^{\prime} \dot{\beta}\right) B+\left(\dot{\beta}-\frac{3}{2} \dot{\alpha}\right) B^{\prime}+\alpha^{\prime} \dot{B}-\frac{1}{2} \dot{B}^{\prime}\right]\right)_{, i}, \\
\delta G_{i}^{0}= & e^{-2 \beta}\left(-(2 \dot{\alpha}+\dot{\beta}) \Phi-(\dot{\alpha}-\dot{\beta}) N+\dot{N}-2 \dot{\Psi}+e^{-2 \beta}\left[-\left(3 \alpha^{\prime 2}+\alpha^{\prime \prime}-2 \alpha^{\prime} \beta^{\prime}\right) B-\left(\beta^{\prime}-\frac{1}{2} \alpha^{\prime}\right) B^{\prime}+\frac{1}{2} B^{\prime \prime}\right]\right)_{, i}, \\
\delta G_{j}^{i}= & e^{-2 \beta} \delta_{j}^{i}\left(\Phi^{\prime \prime}+\left(4 \ddot{\alpha}+2 \ddot{\beta}+6 \dot{\alpha}^{2}\right) \Phi+(\dot{\beta}+2 \dot{\alpha}) \dot{\Phi}+\left(\beta^{\prime}+2 \alpha^{\prime}\right) \Phi^{\prime}-\ddot{N}-\left(4 \alpha^{\prime \prime}+2 \beta^{\prime \prime}+6 \alpha^{\prime 2}\right) N-\left(\beta^{\prime}+2 \alpha^{\prime}\right) N^{\prime}\right. \\
& \left.-(\dot{\beta}+2 \dot{\alpha}) \dot{N}+2 \ddot{\Psi}+6 \dot{\alpha} \dot{\Psi}-6 \alpha^{\prime} \Psi^{\prime}-2 \Psi^{\prime \prime}\right)+\nabla^{2}\left(e^{-2 \alpha}(\Phi+N-\Psi)+e^{-2 \beta}\left[e^{-2 \alpha}(\dot{B}+\dot{\alpha} B)\right.\right. \\
& \left.\left.-3 \dot{\alpha} \dot{E}+3 \alpha^{\prime} E^{\prime}-\ddot{E}+E^{\prime \prime}\right]\right) \delta_{j}^{i}-\left(e^{-2 \alpha}(\Phi+N-\Psi)+e^{-2 \beta}\left[e^{-2 \alpha}(\dot{B}+\dot{\alpha} B)-3 \dot{\alpha} \dot{E}+3 \alpha^{\prime} E^{\prime}-\ddot{E}+E^{\prime \prime}\right]\right)_{, j}^{i},
\end{aligned}
$$

where we denote $h^{\prime}=\partial h / \partial y$ and $\dot{h}=\partial h / \partial t$.

\section{Einstein equation and junction conditions}

In this subsection, we derive the junction conditions. The Einstein equation is given by

$$
\delta G_{N}^{M}=\kappa^{2} e^{-\beta}\left(\delta T_{N}^{M}-N T_{N}^{M}\right) .
$$

We take for the 5D energy-momentum tensor

$$
\delta T_{N}^{M}=\left(\begin{array}{ccc}
0 & 0 & 0 \\
0 & -\delta \rho & (\rho+p) e^{-\alpha_{0}} v_{, i} \\
0 & -(\rho+p) e^{\alpha_{0}} v_{, i} & \delta p \delta_{i j}
\end{array}\right) \delta(y) .
$$

Equating the coefficients of $\delta(y)$ in the Einstein equation gives the following junction conditions:

$$
\begin{aligned}
& \Psi_{1}=-\alpha_{1} N_{0}+\frac{1}{6} \kappa^{2} \delta \rho, \\
& \Phi_{1}=\beta_{1} N_{0}+\kappa^{2}\left(\frac{\delta \rho}{3}+\frac{\delta p}{2}\right), \\
& B_{1}=-2\left(\beta_{1}-\alpha_{1}\right) e^{\alpha_{0}} v, \\
& E_{1}=0,
\end{aligned}
$$

where we use $e^{\beta_{0}}=1$.

[1] V. A. Rubakov and M. E. Shaposhnikov, Phys. Lett. 125B, 136 (1983).

[2] K. Akama, in Gauge Theory and Gravitation, Proceedings, Nara, 1982, Lecture Notes in Physics Vol. 176, edited by K.
Kikkawa, N. Nakanishi, and H. Nariai (Springer-Verlag, Berlin, 1983), pp. 267-271, hep-th/0001113.

[3] L. Randall and R. Sundrum, Phys. Rev. Lett. 83, 4690 (1999); 83, 3370 (1999). 
[4] H. A. Chamblin and H. S. Reall, Nucl. Phys. B562, 133 (1999).

[5] N. Kaloper, Phys. Rev. D 60, 123506 (1999).

[6] T. Nihei, Phys. Lett. B 465, 81 (1999).

[7] H. B. Kim and H. D. Kim, Phys. Rev. D 61, 064003 (2000).

[8] P. Binétruy, C. Deffayet, U. Ellwanger, and D. Langlois, Phys. Lett. B 477, 285 (2000).

[9] E. Flanagan, S. Tye, and I. Wasserman, hep-th/9910498.

[10] P. Kraus, J. High Energy Phys. 12, 011 (1999).

[11] D. Vollick, hep-th/9911181.

[12] S. Mukohyama, Phys. Lett. B 473, 241 (2000).

[13] D. Ida, gr-qc/9912002.

[14] S. Mukohyama, T. Shiromizu, and K. Maeda, Phys. Rev. D 62, 024028 (2000).

[15] J. M. Bardeen, Phys. Rev. D 22, 1882 (1980).

[16] V. F. Mukhanov, H. A. Feldman, and R. H. Brandenberger, Phys. Rep. 215, 203 (1992).

[17] H. Kodama and M. Sasaki, Int. J. Mod. Phys. A 1, 265 (1986).

[18] T. Shiromizu, K. Maeda, and M. Sasaki, Phys. Rev. D 62,
$024012(2000)$

[19] A. Ishibashi and H. Ishihara, Phys. Rev. D 56, 3446 (1997).

[20] J. Garriga and T. Tanaka, Phys. Rev. Lett. 84, 2778 (2000).

[21] T. Tanaka and X. Montes, Nucl. Phys. B582, 259 (2000).

[22] M. Sasaki, T. Shiromizu, and K. Maeda, Phys. Rev. D 62, 024008 (2000).

[23] S. Giddings, E. Katz, and L. Randall, J. High Energy Phys. 03, 023 (2000).

[24] R. Maartens, D. Wands, B. Bassett, and I. Hear, Phys. Rev. D 62, 041301 (2000).

[25] S. W. Hawking, T. Hertog, and H. S. Reall, Phys. Rev. D 62 , 043501 (2000).

[26] S. Kobayashi, K. Koyama, and J. Soda, hep-th/0009160.

[27] H. Kodama, A. Ishibashi, and O. Seto, Phys. Rev. D 62, 064022 (2000).

[28] R. Maartens, Phys. Rev. D 62, 084023 (2000).

[29] D. Langlois, Phys. Rev. D (to be published), hep-th/0005025.

[30] C. van de Bruck, M. Dorca, R. Brandenberger, and A. Lukas, Phys. Rev. D (to be published), hep-th/0005032. 